ARTICLE

\title{
Moiré metrology of energy landscapes in van der Waals heterostructures
}

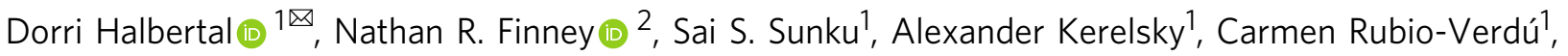 \\ Sara Shabani ${ }^{1}$, Lede Xian (10 3,9 ${ }^{3}$, Stephen Carr ${ }^{4,10}$, Shaowen Chen (1) 1,11, Charles Zhang ${ }^{1,12}$, Lei Wang (1) 1,13, \\ Derick Gonzalez-Acevedo 1,11, Alexander S. McLeod (10) 1, Daniel Rhodes ${ }^{1,14}$, Kenji Watanabe (i) ${ }^{5}$, \\ Takashi Taniguchi ${ }^{5}$, Efthimios Kaxiras (1) ${ }^{6}$, Cory R. Dean (1) ${ }^{1}$, James C. Hone (1) ${ }^{2}$, Abhay N. Pasupathy (1) ${ }^{1}$, \\ Dante M. Kennes (1) ${ }^{3,7}$, Angel Rubio (i) ${ }^{3,8} \&$ D. N. Basov (iD ${ }^{1}$
}

The emerging field of twistronics, which harnesses the twist angle between two-dimensional materials, represents a promising route for the design of quantum materials, as the twistangle-induced superlattices offer means to control topology and strong correlations. At the small twist limit, and particularly under strain, as atomic relaxation prevails, the emergent moiré superlattice encodes elusive insights into the local interlayer interaction. Here we introduce moiré metrology as a combined experiment-theory framework to probe the stacking energy landscape of bilayer structures at the $0.1 \mathrm{meV} /$ atom scale, outperforming the gold-standard of quantum chemistry. Through studying the shapes of moiré domains with numerous nano-imaging techniques, and correlating with multi-scale modelling, we assess and refine first-principle models for the interlayer interaction. We document the prowess of moiré metrology for three representative twisted systems: bilayer graphene, double bilayer graphene and $\mathrm{H}$-stacked $\mathrm{MoSe}_{2} / \mathrm{WSe}_{2}$. Moiré metrology establishes sought after experimental benchmarks for interlayer interaction, thus enabling accurate modelling of twisted multilayers.

\footnotetext{
${ }^{1}$ Department of Physics, Columbia University, New York, NY, USA. ${ }^{2}$ Department of Mechanical Engineering, Columbia University, New York, NY, USA.

${ }^{3}$ Max Planck Institute for the Structure and Dynamics of Matter and Center Free-Electron Laser Science, Luruper. Chaussee 149, 22761 Hamburg, Germany.

${ }^{4}$ Department of Physics, Harvard University, Cambridge, MA 02138, USA. ${ }^{5}$ National Institute for Material Science, Tsukuba, Japan. ${ }^{6}$ John A. Paulson School of Engineering and Applied Sciences, Harvard University, Cambridge, MA 02138, USA. ${ }^{7}$ Institut fur Theorie der Statistischen Physik, RWTH Aachen University, 52056 Aachen, Germany. ${ }^{8}$ Center for Computational Quantum Physics, Flatiron Institute, New York, NY 10010, USA. ${ }^{9}$ Present address: Songshan Lake Materials Laboratory, Dongguan, Guangdong 523808, China. ${ }^{10}$ Present address: Brown University, Providence, RI 02912, USA. ${ }^{11}$ Present address: Department of Physics, Harvard University, Cambridge, MA 02138, USA. ${ }^{12}$ Present address: Department of Physics, University of California at Santa Barbara, Santa Barbara, CA 93106, USA. ${ }^{13}$ Present address: National Laboratory of Solid-State Microstructures, School of Physics and Collaborative Innovation Center of Advanced Microstructures, Nanjing University, Nanjing, China. ${ }^{14}$ Present address: Department of Materials Science and Engineering, University of

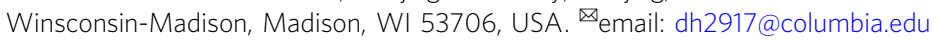


T wisted van der Waals structures, such as twisted bilayer graphene $^{1-16}$ (TBG), twisted double bilayer graphene ${ }^{17-19}$ (TDBG), and twisted transition-metal-dichalcogenides ${ }^{20-27}$ are in the vanguard of quantum materials research ${ }^{28-30}$. The twist between the layers leads to large-scale periodic perturbations of stacking configurations, called a moiré superlattice. Because atomic layers in van der Waals (vdW) materials are not rigid but instead behave as deformable membranes, moiré suprelattices acquire additional attributes. As two atomic layers with a small relative twist angle come in contact, the atomic positions relax to minimize the total energy. Through the relaxation process domains of lowest-energy configurations form and become separated by domain walls of transitionary configurations ${ }^{31-33}$ (Fig. 1a). The generalized stacking fault energy function (GSFE), which provides the energetic variations across different stacking configurations, is the fundamental property that describes relaxed vdW interfaces ${ }^{31,34}$. The GSFE is commonly calculated using density functional theory (DFT) ${ }^{31,34}$. Experimental techniques ${ }^{35}$ to probe the GSFE are currently restricted to the stable lowestenergy configuration, and are very limited in energy resolution compared to the variability among theoretical descriptions.

Here we show that the generalized stacking fault energy function (GSFE) is encoded in fine details of the relaxed moiré super-lattice patterns at the low twist-angle limit. In particular, the shape of domains and domain walls networks, as well as domain wall width, abide by transitionary configurations beyond the lowest-energy stackings of the domains. More specifically, we distinguish between single and double domain walls (SDW and DDW). SDWs separate two distinct stacking configurations of a moiré superlattice (for instance, $\mathrm{ABCA}\left[\mathrm{MM}^{\prime}\right]$ and $\mathrm{ABAB}[\mathrm{MX}$ '] in the TDBG [for twisted $\mathrm{H}$-stacked $\mathrm{MoSe}_{2} / \mathrm{WSe}_{2}$, or T-H$\mathrm{MoSe}_{2} / \mathrm{WSe}_{2}$ for short] example of Fig. 1a). DDWs, formed from the collapse of two SDWs, separate identical phases (ABAB for TDBG and MX' for T-H-MoSe $/ \mathrm{WSe}_{2}$ in Fig. 1a). The formation and nature of DDWs result from attraction of SDWs as they are brought together (for instance, due to external or relaxation induced strain), and is proven here to provide a reliable read-out of the underlying energetics. In cases of inequivalent two lowestenergy configurations (as in Fig. 1), the SDW develops a finite curvature $\kappa$, allowing one to extract the domains energy imbalance with an accuracy outperforming the $\sim 3 \mathrm{meV} /$ atom of the gold standard of quantum chemistry 36,37 .

\section{Results}

Moiré metrology, presented here, correlates measurable spatial patterns of the relaxed moiré superlattice (such as shapes of domains, SDWs and formation of DDWs) with modeling based on the GSFE. To do so, we developed a continuous twodimensional relaxation simulation. The model searches for local interlayer displacement fields that minimize the total energy of the multilayer, as a sum of elastic and stacking energy terms (see Supplementary Information S1-2 for more details, also see ref. ${ }^{33}$ for an alternative approach). The equations are solved in real space and thus capture subtle experimental details that remained underexplored. Fig. 1b-g is a tour-de-force of moiré metrology combining experimental imaging of different systems, techniques and length-scales (Fig. 1b-d), and their respective modeling

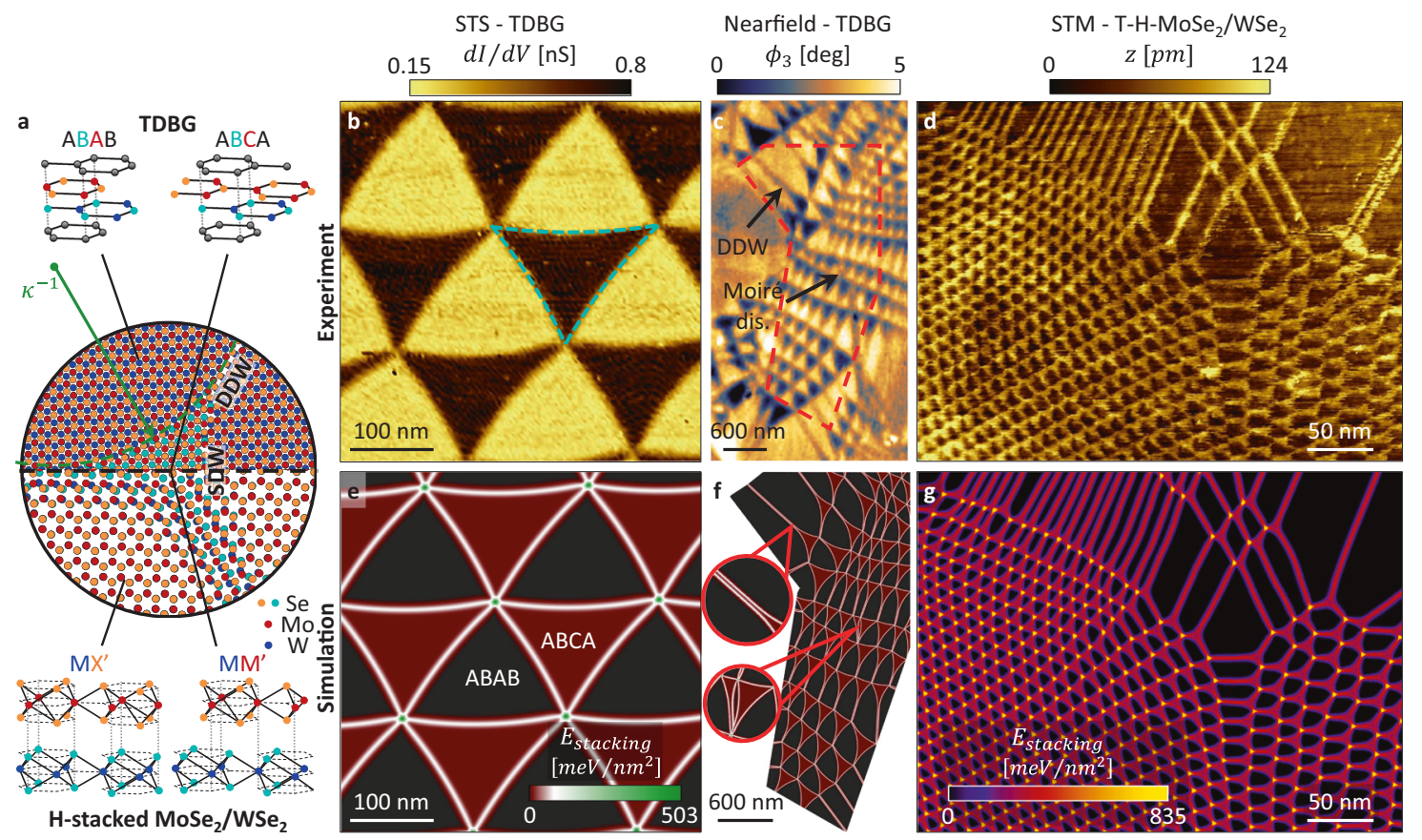

Fig. 1 Physics of atomic layers stacking probed by moiré metrology in vdW twisted bilayers-experiment and theory. a Illustration of domain formations in a relaxed twisted bilayer structure. Center: atomic positioning after relaxation (see Supplementary Information S1 for more details). Atoms are colored to highlight stacking configurations. The energy imbalance leads to curved single domain walls (SDWs), with radius of curvature indicated by $\kappa^{-1}$, and in some cases with formation of double domain walls (DDWs). Two systems with energy imbalance are considered: TDBG (top) and T-H-MoSe $/$ WS $e_{2}$ (bottom). b STS map of TDBG with $\theta=0.07^{\circ}$, revealing rhombohedral ( $A B C A$-dark) and Bernal ( $A B A B$-bright) domains with minimal external strain. The rhombohedral phase bends inward (dashed turquoise line) revealing an energy imbalance between the two phases as discussed in the text. c Mid-IR ( 940 $\mathrm{cm}^{-1}$ ) nearfield phase imaging of TDBG resolves ABCA (dark) and ABAB (bright) phases and DDW formations. d STM map of T-H-MoSe $2 /$ WSe 2 resolving $\mathrm{MM}^{\prime}$ (bright) and $\mathrm{MX}^{\prime}$ (dark) stacking configurations as well as DDW formation in various strain conditions. e- $\mathbf{g}$ Stacking energy density from full relaxation calculations of the experimental cases of (b-d), respectively (see "Methods", Supplementary Information S1-2 and text for more details). The color-map is shared for (e, f). Magnified regions in $\mathbf{f}$ (and arrows in $\mathbf{c}$ ) highlight a DDW formation and a moiré dislocation (see Discussion in Supplementary Information S3). Calculated region of (f) is marked by dashed shape in (c). 
(Fig. 1e-g). Fig. 1b-d were acquired with modern scanning probe microscopy (SPM) techniques: scanning tunneling microscopy (constant current mode) and spectroscopy ${ }^{17}$ (STM and STS respectively) and mid-infrared range (mid-IR) scanning nearfield optical microscopy ${ }^{11}$ (SNOM). These techniques resolve stacking configurations based on local topographic and electronic (STM and STS), as well as mid-IR optical conductivity (mid-IR SNOM) contrasts. In low strain TDBG, the model (Fig. 1e) captures the fine curving of SDWs (Fig. 1b). In cases of higher strain (Fig. 1c and modeling in Fig. 1f) we observe the formation of one dimensional DDW structures (inset of Fig. If highlights an example). Similarly, DDW formations and SDW curving were observed (Fig. 1d) and modeled (Fig. 1g) in T-H-MoSe $2 / \mathrm{WSe}_{2}$, with excellent agreement across different length-scales of the image (see Supplementary Information S3 for additional analysis). Next, we will illustrate in detail how moiré super-lattices reveal the energy landscape information using TBG and TDBG as prototypical examples.

Moiré metrology of twisted bilayer graphene. To study the energy landscape of TBG, we focus on the interplay between SDW and DDW formations. Fig. 2a presents a non-local nanophotocurrent map of TBG in the minimal twist limit $<0.1^{\circ}$. Bright spots in the photo-current map highlight the AA sites (indicating higher absorption-see "Methods" and Supplementary Information S4). The AA sites are connected by domain walls separating $\mathrm{AB}$ and $\mathrm{BA}$ domains. The resultant moiré super-lattice is clearly affected by strain, inferred from the distorted triangular pattern, especially near the edges of the stack. There, we observe the merging of two SDWs into a single DDW (selected locations are marked in Fig. 2a). We successfully account for the observed network within a model addressing a competition between SDWs and DDWs. To grasp the essential physics, we first assume a characteristic energy of forming a segment of DDW and SDW. We define a dimensionless domain-wall formation ratio as the ratio of $\mathrm{DDW}$ and SDW line energies, $\bar{\beta}=\gamma_{D D W} / \gamma_{S D W}$. In addition to $\bar{\beta}$, the model input includes the AA sites of the moiré pattern as the fixed vertices of the triangles forming the network. We explore the SDW vs. DDW structures that emerge for a given value of the single tuning parameter $\bar{\beta}$. The case of $\bar{\beta}=2$ implies there is no benefit in forming a DDW, and the optimal structure would simply be straight SDWs connecting the AA sites. For $\bar{\beta}<2$ the two SDWs attract each other favoring the emergence of DDW segments (see Supplementary Information S4 and Supplementary Video 1 for details). Our modeling captures the overall shape of the experimental map for $\bar{\beta}=1.90$ (Fig. 2a). The agreement is remarkable considering the minimal modeling we employ. We conclude that in order for a TBG model to reproduce the experimental picture, two SDWs have to sufficiently attract one another as quantified by the fitted $\bar{\beta}$. In that sense, as we show more rigorously below, moiré metrology puts constraints the GSFE.

To quantify how the observed moiré networks constrain the stacking energy landscape, we span all realistic GSFE's satisfying the symmetry of TBG over a 2D unit-less parameter space $(\zeta, \tau)$ (as illustrated in Fig. $2 \mathrm{~b}$ and discussed at Supplementary Information S4), such that each point on the $(\zeta, \tau)$ plane represents one GSFE candidate. We solve a set of $1 \mathrm{D}$ relaxation problems describing the profiles of SDWs and DDWs (see Supplementary Information S1 for more details on relaxation codes), and extract the domain wall formation ratio $\bar{\beta}$. This allows us to define a band in $(\zeta, \tau)$ plane of GSFE's that comply with the
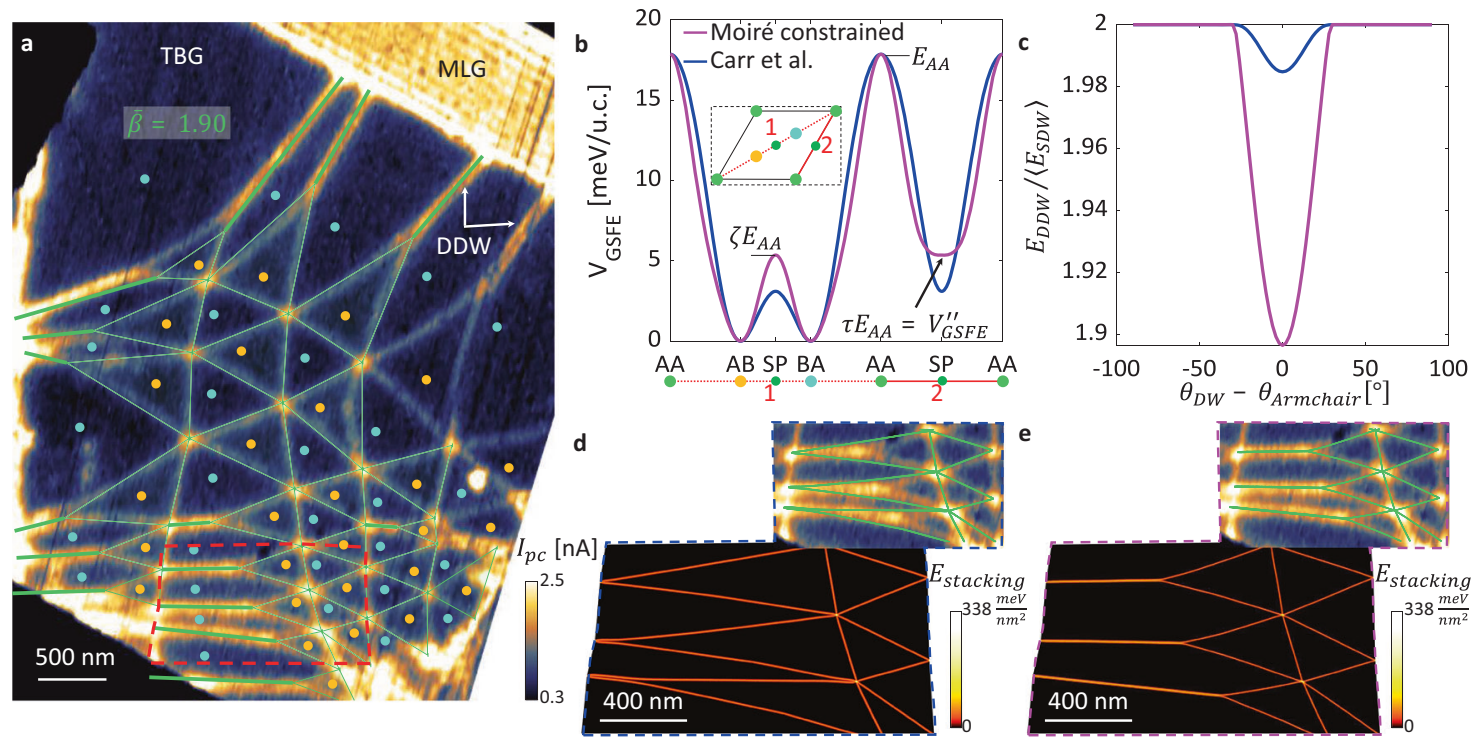

Fig. 2 Energy landscape of twisted bilayer graphene (TBG) revealed by the interplay between double (DDW) and single (SDW) domain walls. a Nonlocal nano-photocurrent map of moire super-lattice of a TBG sample at the minimal twist limit (see "Methods" for more details). The technique reveals the formation of DDWs (marked by "DDW") at strained domains, separating domains of identical stacking configurations (each configuration is indicated by dots of a given color $[A B-$ orange, $B A-c y a n]$ ). The green network overlaid on the data corresponds to the prediction by a single tuning parameter model with $\bar{\beta}=1.90$ (see text and Supplementary Information S6). b GSFE of TBG from ref. ${ }^{31}$ (blue) and a moiré constrained version (magenta). The unit-less parameters $\zeta$, $\tau$, spanning the phase space of GSFE candidates for TBG, are illustrated (see Supplementary Information S6). Inset: path in configuration space for presented GSFE line-cuts. c Effective attraction between SDWs as reflected by DDW to SDW energy ratios for different SDW orientations (relative to armchair direction) for both models. $E_{\mathrm{DDW}}$ is the DDW line-energy for a DDW along the armchair direction and similarly $E_{\mathrm{SDW}}$ is for the average of the two SDWs comprising the DDW. d, e Stacking energy density from 2D relaxation calculation (see "Methods" and Supplementary Information S2) for the two discussed GSFE choices ( $\mathbf{d}$-literature, $\mathbf{e}$-moiré constrained version with $\tau=0.025, \zeta=0.3$ ) of region marked by red dashed frame in (a), showing fundamental differences in formation of DDWs. Inset: extracted domain wall structures from relaxation calculations overlaid on experimental results. 
experimental $\bar{\beta}=1.90$ (see Supplementary Fig. S3g). Fig. $2 b$ compares one GSFE moiré constrained candidate with the $\bar{\beta}=$ 1.90 band (magenta) with the well-accepted choice of GSFE of ref. 31 (blue), which notably falls outside of the band with $\bar{\beta}=1.98$. The moiré metrology analysis indicates that SDWs implied by GSFE in ref. ${ }^{31}$ insufficiently attract one another (blue curve in Fig. 2c) to account for the observed network, as indeed revealed in Fig. 2 d. In contrast, the moiré-constrained candidate (magenta in Fig. 2b) with a flatter saddle point promotes stronger SDWs attraction across a broad range of domain wall orientations (magenta curve in Fig. 2c; see Supplementary Information S1 and S5 for additional details), and yields excellent agreement with the data (Fig. 2e). Regardless of the good agreement, the relatively flat saddle point comes as a surprise, and may in fact correct for an unknown effect unrelated to interlayer energy.

Moiré metrology of twisted double bilayer graphene. Compared to TBG, the TDBG system makes an even more interesting casestudy due to the small yet finite imbalance between the two lowest-energy phases: Bernal (ABAB) and rhombohedral (ABCA) stackings ${ }^{17}$. This imbalance results in an energy cost per-unitarea $(\sigma)$ for rhombohedral relative to Bernal stackings, leading to characteristic curved domains ${ }^{17,38}$ (see Fig. 1a, b). Exploring large areas of TDBG reveals a rich distribution of rhombohedral domain shapes (see Fig. 1c and other TDBG images in this work). Fig. 3a summarizes this distribution as a histogram of inverse curvature values $\left(\kappa^{-1}\right)$, extracted from images as in Fig. $1 \mathrm{~b}$ (see Supplementary Information S6 for more examples). The histogram reveals a distinct clustering about a value of $\kappa^{-1}=440 \pm$ $120 \mathrm{~nm}$, which we use to assess the accuracy of several variants of the GSFE from available DFT functionals (Fig. $3 \mathrm{~b}$ and see "Methods"). All reported GSFE variants are qualitatively similar to the TBG case, peaking at the BAAC configuration, and having a saddle point barrier between $\mathrm{ABAB}$ and $\mathrm{ABCA}$. A closer inspection (inset) reveals a profound difference between the GSFEs for the ABCA relative to the $\mathrm{ABAB}$ that governs domain curvature. We model the domain curvature and structure by a continuous 2D relaxation code (Supplementary Information S1). Fig. 3c, d show two representative cases, with disparate outcomes. In Fig. 3c (resembling the experimental case of Fig. 1b) the energy is minimized by slight bending of the SDW into the ABCA region. As the twist angle decreases (or as strain increases as in Fig. 1c), at some point it becomes energetically beneficial to form DDWs (Fig. 3d). As the twist angle further decreases, the shape of the ABCA domains remains unchanged. Similarly, solving for the domain formation for all DFT approaches and across a wide twist angle range, we compare the extracted $\kappa^{-1}$. Interestingly, $\kappa$ is independent of the twist angle for all GSFE variants (with values indicated by colored lines over Fig. 3a), which is not generally the
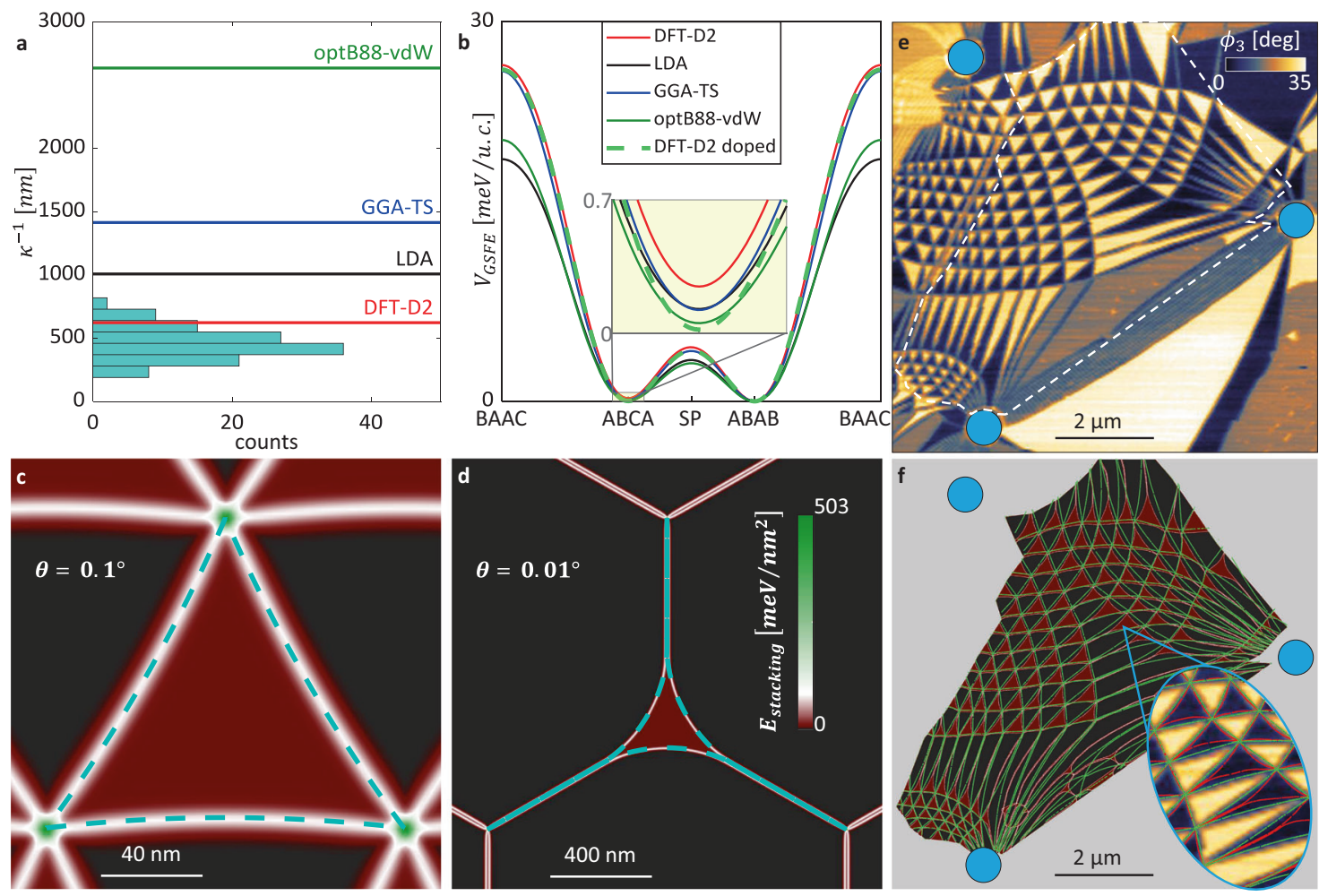

Fig. 3 Moiré super-lattice study of rhombohedral domains in twisted double bilayer graphene (TDBG). a Curvature histogram across all measured domains near charge neutrality point (see discussion in Supplementary Information S7), showing a clear cluster at $440 \pm 120 \mathrm{~nm}$. Calculated curvatures of 4 DFT approaches (of $\mathbf{b}$ ) are illustrated over the histogram (colored horizontal lines). b GSFE of TDBG based on four different approaches (solid lines, see "Methods"). The dashed light-green line is the GSFE for DFT-D2 approach at a doping level of $8 \times 10^{12} \mathrm{~cm}^{-2}$. Inset: enlarged view highlighting small difference between $A B C A$ and $A B A B$ configurations $\left(V_{G S F E}(A B A B)=0\right.$ identically). c, d Mechanical relaxation solutions (false-color: stacking energy density) and "soap-bubble" model domain shape (dashed turquoise) for two representative twist angles (c: $\left.\theta=0.1^{\circ}, \mathbf{d}: \theta=0.01^{\circ}\right)$ for DFT-D2. e Mid-IR $\left(940 \mathrm{~cm}^{-1}\right.$ ) nearfield phase imaging of a defect-induced doped TDBG (see Supplementary Information S7). 3 holes punctured one of the bilayers (blue circles-see "Methods") and induce a non-trivial external strain map. Mid-IR imaging resolves ABCA (dark) and ABAB (bright) phases and double-domain wall (DDW) formations (for instance, the multiple-DDW formation connecting bottom holes). $\mathbf{f}$ Comparing relaxation calculations solutions of un-doped vs. $8 \times 10^{12} \mathrm{~cm}^{2}$ doped DFT-D2 approach GSFE, simulating the experimental case (marked by dashed shape in e), False-color represents stacking energy density of un-doped case, overlaid (green dots) with tracked domain walls in the doped case. Inset: highlighting differences between model by overlaying domain wall formation of doped (red) and un-doped (green) cases over strained region in the experimental map. The color-map is shared for (c-e). 
case (see discussion in Supplementary Information S7). The domain structures are further captured by the 2D "soap-bubble" model, as seen in turquoise dashed lines in the representative cases of Fig. 3c, d and more generally in Supplementary Videos 2-5. This model approximates the total energy as a sum of a domain area term and two line-energy terms as $E=\int_{S D W} d l \gamma_{1}(\varphi)+\int_{D D W} d l \gamma_{2}(\varphi)+\sigma S$, where $\gamma_{1,2}$ are the lineenergies of SDW and DDW as a function of the domain-wall orientation respectively, the integrations are along the domain walls, and $S$ is the area of the domain (see Supplementary Information S7). All model parameters require only the GSFE (and elastic properties) to describe domain shapes, with no additional tuning parameters (Supplementary Information S7). One approach, DFT-D2, remarkably reproduces the experimental cluster (Fig. 3a), due to relatively high $\sigma$ and comparable lineenergies to other approaches (see Supplementary Information S2). It is noteworthy that the DFT-D approach has not been previously considered as the leading approach when theoretically benchmarked against the Quantum Monte-Carlo method for the binding energy of $\mathrm{AA}$ and $\mathrm{AB}$ stacking of bilayer graphene ${ }^{39}$.

The rhombohedral domains represented in the histogram of Fig. 3a exemplify a well-defined electrostatic environment near charge neutrality point (CNP) in the absence of the interlayer bias. As shown recently ${ }^{38}$, upon charging and biasing the balance between the rhombohedral and Bernal phases can shift. An extreme demonstration of malleability of TDBG moiré patterns under a non-uniform distribution of charges and high strain conditions is presented in Fig. 3e. Three holes (marked by blue circles) punctured one of the bilayers. This procedure prompts a highly strained moiré pattern, most strongly manifested in the densely packed parallel DDWs structures connecting the two bottom holes. The stack shows strong defect-induced doping (see discussion in Supplementary Information S5), apparent in the enhanced nearfield contrast between the ABCA (dark) and ABAB (bright) phases (compare to contrast of Fig. 1c). Further support for the high non-uniformity of charge distribution is an observed region of flipped balance, where the $\mathrm{ABAB}$ phase becomes unstable relative to $\mathrm{ABCA}$ across a sharp $(\sim 50 \mathrm{~nm})$ interface (to the left of the top hole). Attempting to model the moiré superlattice with the DFT-D2 GSFE at CNP (red in Fig. 3b) fails to capture the observed structure of excessively curved SDWs (color-map of Fig. 3f). However, when introducing a doping level of $8 \times 10^{12} \mathrm{~cm}^{-2}$ the resulting GSFE (dashed light-green in Fig. $3 \mathrm{~b}$ ) better captures the observed structure (green dots in Fig. $3 \mathrm{f}$ tracking the domain walls in the calculation). The difference between the two models becomes more pronounced for regions of higher strain, as highlighted in the inset of Fig. $3 \mathrm{f}$ (compare green and red dots in respect to the experimental map). Therefore, minuscule energy differences between models of order $0.1 \mathrm{meV} /$ u.c. (inset of Fig. 3b) result in measurable spatial features of the relaxed moiré patterns. To put this figure in context, the theoretical method which is widely considered as the goldstandard of ab-initio quantum chemistry ${ }^{36,37}$ yields an accuracy as low as $3 \mathrm{meV} /$ atom $^{37}$.

\section{Discussion}

To understand the enhanced sensitivity of moiré metrology under strain (as seen in Fig. 3f), we propose an alternative description of the moiré superlattice in terms of a geometric interference pattern of the lattices of the two layers (see Supplementary Information S1). At minute twist angles, the relaxed moiré patterns are essentially a projection of the detailed energy landscape over space, accumulated over large regions compared to the atomic scale. The introduction of strain between the layers, whether naturally occurring or externally controlled, alters the interference pattern (Supplementary Information S8). As strain pushes the domain walls in Figs. 1-3 together, it also promotes their interaction; both effects are reflected in the relaxed moiré pattern (also see Supplementary Video 6).

Moiré metrology, introduced here, correlates first principle calculations of the stacking energy function with measurable spatial features of twisted vdW systems. The stacking energy function is widely used for modeling twisted multilayers across a broad range of twist angles and strain conditions, and has direct implications for the electronic band-structure ${ }^{40}$. Moiré metrology is not restricted to the discussed material systems and can be universally applied with other systems of great current interest ${ }^{41-48}$. Therefore, by providing a reliable account of the stacking energy function, moiré metrology has a broad impact across the field of vdW heterostructures. Furthermore, the moiré metrology tools can also be used for modeling and designing non-uniform strain fields in realistic devices. Finally, due to its outstanding stacking energy sensitivity, we propose moiré metrology as a concrete experimental path to provide much needed benchmarks for first-principle theoretical approaches ${ }^{49}$.

\section{Methods}

\section{Samples preparation}

Source crystals. The bulk crystals of $\mathrm{MoSe}_{2}$ and $\mathrm{WSe}_{2}$ were grown by the self-flux method $^{50}$ : single crystals of $\mathrm{MoSe}_{2}$ were prepared by combining Mo powder (99.997\%, Alfa Aesar 39686) and Se shot (99.999\%, Alfa Aesar 10603) in a ratio of 1:50 (Mo:Se) in a quartz ampoule. The ampoules were subsequently sealed under vacuum $\left(\sim 5 \times 10^{-6}\right.$ Torr $)$. The reagents were then heated to $1000^{\circ} \mathrm{C}$ within $24 \mathrm{~h}$ and dwelled at this temperature for 8 weeks before being cooled to $350^{\circ} \mathrm{C}$ over 4 weeks. At $350^{\circ} \mathrm{C}$ the Se flux was decanted through alumina wool (Zircar D9202) in a centrifuge and the ampoules were quenched in air. The subsequently obtained $\mathrm{MoSe}_{2}$ single crystals were annealed at $275^{\circ} \mathrm{C}$ with a $200^{\circ} \mathrm{C}$ gradient for $48 \mathrm{~h}$ to remove any residual Se. A similar process was also used to synthesize single crystals of $\mathrm{WSe}_{2}$ with 1:15 (W:Se) as the starting ratio and using W powder $(99.999 \%$, Alfa Aesar 12973). Kish graphite source crystals were purchased from Graphene Supermarket.

Exfoliation. The $\mathrm{MoSe}_{2}$ and $\mathrm{WSe}_{2}$ monolayers, and Graphene and hBN flakes were mechanically exfoliated from the bulk single crystals onto $\mathrm{SiO}_{2} / \mathrm{Si}(285 \mathrm{~nm}$ oxide thickness) chips using the tape-assisted exfoliation technique (the tape used was Scotch Magic Tape). The exfoliation followed ref. ${ }^{51}$, such that the Si chips were treated with $\mathrm{O}_{2}$ plasma (using a benchtop radio frequency oxygen plasma cleaner of Plasma Etch Inc., PE-50 XL, $100 \mathrm{~W}$ at a chamber pressure of $\sim 215 \mathrm{mTorr}$ ) for $20 \mathrm{~s}$ for graphene, for $10 \mathrm{~s}$ for $\mathrm{MoSe}_{2}$ and $\mathrm{WSe}_{2}$, and no $\mathrm{O}_{2}$ plasma treatment for $\mathrm{hBN}$. The chips were then matched with respective exfoliation tape. In the graphene case the chip+tape assembly were heated at $100^{\circ} \mathrm{C}$ for $60 \mathrm{~s}$ and cooled to room temperature prior to removing the tape. Such thermal treatment was not done for other materials. The $\mathrm{MoSe}_{2}$ and $\mathrm{WSe}_{2}$ monolayer relative crystallographic orientation was obtained by linear-polarization- resolved second-harmonic generation (SHG).

Stack preparation. All heterostructures were assembled using standard dry-transfer techniques $^{52}$ with a polypropylene carbonate (PPC) film mounted on a

transparent-tape-covered polydimethylsiloxane (PDMS) stamp. The transparent tape layer was added to the stamp to mold the PDMS into a hemispherical shape which provides precise control of the PPC contact area during assembly ${ }^{53}$.

All graphene heterostructures were made by first picking up the bottom-layer boron nitride (h-BN) ( $>25 \mathrm{~nm}$ thick), followed in the case of the TDBG samples by a graphite bottom gate-layer ( $>5 \mathrm{~nm}$ thick), then a dielectric $\mathrm{BN}$ layer $(>25 \mathrm{~nm}$ thick). Prior to pick-up, mechanically exfoliated graphene flakes on $\mathrm{Si} / \mathrm{SiO}_{2}$ were separately patterned with anodic-oxidation lithography ${ }^{54}$ to facilitate the "cut-andstack" technique ${ }^{55}$ where it was used (all samples but those used for STM, which used the established tearing method). In the case of the TDBG samples, additional anodic-oxidation lithography was used prior to pick-up to provide additional texture to the strain landscape, e.g., cut holes inside the bulk of one of the graphene layers or non-rectilinear edge geometries.

In the case of $\mathrm{MoSe}_{2} / \mathrm{WSe}_{2}$ the PPC was used to pick up a thin layer of exfoliated h-BN and a few layers of graphene. Then a monolayer $\mathrm{WSe}_{2}$ was picked up and using the SHG data $\mathrm{MoSe}_{2}$ monolayer was lifted on a rotation stage with $\sim 1^{\circ}$ twist angle. The stack was flipped over a Si/SiO $(285 \mathrm{~nm})$ chip at $120^{\circ} \mathrm{C}$. In the last step, the sample was thermally annealed in a high vacuum chamber to remove the PPC at $250^{\circ} \mathrm{C}$ for $1 \mathrm{~h}$.

Some of the presented measurements (TDBG stacks of Fig. 3e, Supplementary Figs. S8b, d-f and red bins in histogram of Supplementary Fig. S8a) were taken at this point while the stack was on a PDMS/transparent-tape/PPC structure. This 
provided access to the meta-stable large rhombohedral domain before they were suppressed by thermal annealing (see Supplementary Information S9).

After optional mid-assembly scanning probe measurement and/or optional encapsulation of the twisted-graphene layers, the PPC film with the heterostructure on top is mechanically removed from the transparent-tape-covered PDMS stamp and placed onto a $\mathrm{Si} / \mathrm{SiO}_{2}$ substrate such that the final pick-up layer is the top layer.

In the case of the TBG device presented in this work, the underlying PPC was removed by vacuum annealing at $\mathrm{T}=350^{\circ} \mathrm{C}$. Standard plasma etching and metal deposition techniques ${ }^{52}$ were then used to shape and make contact to the samples. In the case of the TDBG devices for STM (Fig. 1b) the stack was made using the established tearing method, using PPC as a polymer to sequentially pick up hBN, half of a piece of graphene followed by the second half with a twist angle.

For all samples for STM imaging, standard metal deposition techniques were avoided in order to maintain a pristine surface, therefore, direct contact was made to the stack by micro-soldering with Field's metal ${ }^{56}$, keeping temperatures below $80 \mathrm{C}$ during the entire process.

Nearfield imaging techniques. In this work, we used two nano-optical imaging techniques: cryogenic nano-photocurrent imaging (used for TBG imaging) and phase-resolved scattering type scanning optical microscope imaging (used for TDBG imaging) (s-SNOM). Cryogenic photocurrent imaging 57 was done with a home-built cryogenic SNOM, and s-SNOM nearfield imaging was done with a commercial (Neaspec) SNOM ${ }^{11}$. In both cases using mid-IR light (continuous wave $\mathrm{CO}_{2}$ gas laser [Access Laser] at a wavelength of $10.6 \mu \mathrm{m}$ ) focused to a diffraction limited spot at the apex of a metallic tip, while raster scanning the sample at tapping mode. Fig. $2 \mathrm{a}$ was acquired at a temperature of $100 \mathrm{~K}$ and while tuning the silicon back-gate to a relatively high doping of $3 \times 10^{12} \mathrm{~cm}^{-2}$. In such a case, we observe, for the first time, a non-local photo-current generation regime. In this regime, the light induced temperature profile is broad (relative to system size) and the photo-current generation is located at a distant interface (a monolayer twisted bilayer interface in this case, clearly visible as the bright region with plasmonic fringes at the top right section of Fig. 2a). The signal contrast in such a case results from absorption contrast between different stacking configuration, and not from thermo-electric properties. This unique approach provides a high-resolution image, not limited by thermal length-scales (see Supplementary Information S4 for more details).

In the s-SNOM case, we collect the scattered light (power of $3 \mathrm{~mW}$ ) by a cryogenic $\mathrm{HgCdTe}$ detector (Kolmar Technologies). The far-field contribution to the signal can be eliminated from the signal by locking to a high harmonic (here we used the 3rd harmonic of the tapping). The phase of the backscattered signal was extracted using an interferometric detection method, the pseudo-heterodyne scheme, by interfering the scattered light with a modulated reference arm at the detector. Fig. 1c was acquired with a level of $1 \times 10^{12} \mathrm{~cm}^{2} \mathrm{p}$-doping applied with a Si backgate. Such a level of doping has negligible effect on domain curvature (see Supplementary Information S5 for more details).

STM and STS imaging. STM and STS measurements were carried out in a homebuilt STM under ultra-high vacuum conditions. $\mathrm{MoSe}_{2} / \mathrm{WSe}_{2}$ measurements were performed at $300 \mathrm{~K}$ while TDBG measurements were performed at $5.7 \mathrm{~K}$. The setpoints of the STM (constant current mode) imaging were $\mathrm{V}=-1.8 \mathrm{~V}$ and $\mathrm{I}=$ $100 \mathrm{pA}$ for $\mathrm{MoSe}_{2} / \mathrm{WSe}_{2}$ measurements and $\mathrm{V}=0.5 \mathrm{~V}$ and $\mathrm{I}=50 \mathrm{pA}$ for TDBG measurements. The tip-sample bias for the STS measurement of Fig. $1 \mathrm{~b}$ was -57.5 $\mathrm{meV}$. STM tips were prepared and calibrated for atomic sharpness and electronic integrity on freshly prepared $\mathrm{Au}$ (111) crystals. Samples were measured with multiple tips to ensure consistency of results.

Parameter DFT calculations. The DFT calculations of the GSFE parameters were performed with the Vienna Ab initio Simulation Package (VASP) ${ }^{58}$. Plane wave basis sets were employed with energy cutoff of $1200 \mathrm{eV}$ and $500 \mathrm{eV}$ for the calculations for TDBG and $\mathrm{MoSe}_{2} / \mathrm{WSe}_{2}$, respectively. Pseudopotentials were constructed with the projector augmented wave method (PAW) ${ }^{59,60} .60 \times 60 \times 1$ and $11 \times 11 \times 1 \Gamma$-centered k-point grids were used in the calculations for TDBG and $\mathrm{MoSe}_{2} / \mathrm{WSe}_{2}$, respectively. Vacuum spacing larger than $15 \AA$ was added along the $z$ direction to eliminate the artificial interaction between periodic slab images in all the calculations. In the calculations of the GSFE, the $x-y$ coordinates of the atoms in all the 2D layers were fixed and the $z$ coordinates were allowed to relax until forces in the $z$-direction are less than $1 \mathrm{meV} / \AA$ for TDBG and $20 \mathrm{meV} / \AA$ for $\mathrm{MoSe}_{2} / \mathrm{WSe}_{2}$. The van der Waals interactions are important in evaluating the energetics of the 2D layer structures. We tested this effect for TDBG by considering four approaches: (1) employing PAW pseudopotentials with the exchangecorrelation functionals treated at the local density approximation (LDA) level. (2) Employing PAW pseudopotentials with the exchange-correlation functionals treated at the generalized gradient approximation (GGA) level ${ }^{61}$ and additional van der Waals corrections are applied with the DFT-D2 method of Grimme ${ }^{62}$. (3) Employing PAW pseudopotentials with the GGA functionals and additional van der Waals corrections with the Tkatchenko-Scheffler method ${ }^{63}$. (4) Employing PAW pseudopotentials with a non-local correlation functional (optB88-vdW) ${ }^{64-66}$ that approximately accounts for dispersion interactions. In the calculations for $\mathrm{MoSe}_{2} / \mathrm{WSe}_{2}$, we employed PAW pseudopotentials with the GGA PBE functionals with the DFT-D3 van der Waals corrections ${ }^{67}$. The bulk modulus and shear modulus for each material were calculated by applying isotropic or uniaxial strain to a monolayer lattice and then performing a quadratic fit to the strain-dependent energies. For TDBG, we assume the elastic coefficients are twice the values extracted for monolayer graphene. The DFT ground state energy is evaluated on a regular grid of different interlayer configurations. The Fourier components of the resulting energies are then extracted to create a convenient functional form for the GSFE.

\section{Data availability}

The raw datasets used for the presented analysis within the current study are available from the corresponding author on reasonable request.

\section{Code availability}

Developed relaxation codes can be provided from the corresponding author on reasonable request.

Received: 25 September 2020; Accepted: 2 December 2020; Published online: 11 January 2021

\section{References}

1. Lopes Dos Santos, J. M. B., Peres, N. M. R. \& Castro Neto, A. H. Graphene bilayer with a twist: Electronic structure. Phys. Rev. Lett. 99, 19-22 (2007).

2. Bistritzer, R. \& MacDonald, A. H. Moiré bands in twisted double-layer graphene. Proc. Natl Acad. Sci. USA 108, 12233-12237 (2011).

3. Yankowitz, M. et al. Tuning superconductivity in twisted bilayer graphene. Science 363, 1059-1064 (2019).

4. Lu, X. et al. Superconductors, orbital magnets and correlated states in magic angle bilayer graphene. Nature 574, 653-657 (2019).

5. Sharpe, A. L. et al. Emergent ferromagnetism near three-quarters filling in twisted bilayer graphene. Science 365, 605-608 (2019).

6. Zondiner, U. et al. Cascade of phase transitions and Dirac revivals in magic angle graphene. Nature 582, 203-208 (2020).

7. Stepanov, P. et al. Untying the insulating and superconducting orders in magic-angle graphene. Nature 583, 375-378 (2020).

8. Uri, A. et al. Mapping the twist-angle disorder and Landau levels in magicangle graphene. Nature 581, 47-52 (2020).

9. Alden, J. S. et al. Strain solitons and topological defects in bilayer graphene. Proc. Natl Acad. Sci. USA 110, 11256-11260 (2013).

10. Cao, Y. et al. Unconventional superconductivity in magic-angle graphene superlattices. Nature 556, 43-50 (2018).

11. Sunku, S. S. et al. Photonic crystals for nano-light in moiré graphene superlattices. Science 362, 1153-1156 (2018).

12. Huang, S. et al. Topologically Protected Helical States in Minimally Twisted Bilayer Graphene. Phys. Rev. Lett. 121, 37702 (2018).

13. Rickhaus, P. et al. Transport Through a Network of Topological Channels in Twisted Bilayer Graphene. Nano Lett. 18, 6725-6730 (2018).

14. $\mathrm{Xu}, \mathrm{S}$. G. et al. Giant oscillations in a triangular network of one-dimensional states in marginally twisted graphene. Nat. Commun. 10, 1-5 (2019).

15. Hesp, N. C. H. et al. Collective excitations in twisted bilayer graphene close to the magic angle. arXiv 1910.07893. Preprint at https://arxiv.org/abs/ 1910.07893 (2019).

16. Choi, Y. et al. Electronic correlations in twisted bilayer graphene near the magic angle. Nat. Phys. 15, 1174-1180 (2019).

17. Kerelsky, A. et al. Moire-less correlations in ABCA Graphene. arXiv 1911.00007. Preprint at https://arxiv.org/abs/1911.00007 (2019).

18. Shen, C. et al. Correlated states in twisted double bilayer graphene. Nat. Phys. 16, 520-525 (2020)

19. Liu, X. et al. Tunable spin-polarized correlated states in twisted double bilayer graphene. Nature 583, 221-225 (2020).

20. Scuri, G. et al. Electrically tunable valley dynamics in twisted $\mathrm{WSe} \mathrm{S}_{2} / \mathrm{WSe}_{2}$ bilayers. Phys. Rev. Lett. 124, 217403 (2020).

21. Weston, A. et al. Atomic reconstruction in twisted bilayers of transition metal dichalcogenides. Nat. Nanotechnol. 15, 592-597 (2020).

22. Rosenberger, M. R. et al. Twist angle-dependent atomic reconstruction and Moiré patterns in transition metal dichalcogenide heterostructures. ACS Nano 14, 4550-4558 (2020).

23. Wu, F., Lovorn, T., Tutuc, E. \& Macdonald, A. H. Hubbard model physics in transition metal dichalcogenide Moiré bands. Phys. Rev. Lett. 121, 26402 (2018).

24. $\mathrm{Yu}, \mathrm{H}$. et al. Moiré excitons: from programmable quantum emitter arrays to spin-orbit-coupled artificial lattices. Sci. Adv. 3, e1701696 (2017).

25. Wang, J. et al. Diffusivity reveals three distinct phases of interlayer excitons in $\mathrm{MoSe}_{2} / \mathrm{WSe}_{2}$ heterobilayers. arXiv 2001.03812. Preprint at https://arxiv.org/ abs/2001.03812 (2020). 
26. Shimazaki, Y. et al. Strongly correlated electrons and hybrid excitons in a moiré heterostructure. Nature 580, 472-477 (2020).

27. Jin, C. et al. Observation of moiré excitons in WSe2/WS2 heterostructure superlattices. Nature 567, 76-80 (2019).

28. Balents, L., Dean, C. R., Efetov, D. K. \& Young, A. F. Superconductivity and strong correlations in moiré flat bands. Nat. Phys. 16, 725-733 (2020).

29. Kennes, D. M. et al. Moiré heterostructures: a condensed matter quantum simulator. Preprint at https://arxiv.org/abs/2011.12638 (2020).

30. Yankowitz, M., Ma, Q., Jarillo-Herrero, P. \& LeRoy, B. J. van der Waals heterostructures combining graphene and hexagonal boron nitride. Nat. Rev. Phys. 1, 112-125 (2019).

31. Carr, S. et al. Relaxation and domain formation in incommensurate twodimensional heterostructures. Phys. Rev. B 98, 224102 (2018).

32. Cazeaux, P., Luskin, M. \& Massatt, D. Energy minimization of two dimensional incommensurate heterostructures. Arch. Ration. Mech. Anal. 235, 1289-1325 (2020).

33. Enaldiev, V. V., Zólyomi, V., Yelgel, C., Magorrian, S. J. \& Fal'ko, V. I. Stacking domains and dislocation networks in marginally twisted bilayers of transition metal dichalcogenides. Phys. Rev. Lett. 124, 206101 (2020).

34. Zhou, S., Han, J., Dai, S., Sun, J. \& Srolovitz, D. J. Van der Waals bilayer energetics: generalized stacking-fault energy of graphene, boron nitride, and graphene/boron nitride bilayers. Phys. Rev. B 92, 155438 (2015).

35. Wang, W. et al. Measurement of the cleavage energy of graphite. Nat. Commun. 6, 7853 (2015)

36. Dykstra, C. E., Frenking, G., Kim, K. S. \& Scuseria, G. E. Computing technologies, theories, and algorithms: The making of 40 years and more of theoretical and computational chemistry. Theory Appl. Comput. Chem. https:// doi.org/10.1016/B978-044451719-7/50044-5 (2005).

37. Neese, F., Atanasov, M., Bistoni, G., Maganas, D. \& Ye, S. Chemistry and quantum mechanics in 2019: give us insight and numbers. J. Am. Chem. Soc. 141, 2814-2824 (2019).

38. $\mathrm{Li}, \mathrm{H}$. et al. Global control of stacking-order phase transition by doping and electric field in few-layer graphene. Nano Lett. 20, 3106-3112 (2020).

39. Mostaani, E., Drummond, N. D. \& Fal'Ko, V. I. Quantum Monte Carlo calculation of the binding energy of bilayer graphene. Phys. Rev. Lett. 115, 115501 (2015).

40. Carr, S., Fang, S., Zhu, Z. \& Kaxiras, E. Exact continuum model for low-energy electronic states of twisted bilayer graphene. Phys. Rev. Res 1, 013001 (2019).

41. Tong, Q., Liu, F., Xiao, J. \& Yao, W. Skyrmions in the Moiré of van der Waals 2D Magnets. Nano Lett. 18, 7194-7199 (2018).

42. Van Wijk, M. M., Schuring, A., Katsnelson, M. I. \& Fasolino, A. Moiré patterns as a probe of interplanar interactions for graphene on h-BN. Phys. Rev. Lett. 113, 135504 (2014).

43. Woods, C. R. et al. Charge-polarized interfacial superlattices in marginally twisted hexagonal boron nitride. arXiv 2010.06914. Preprint at https://arxiv org/abs/2010.06914 (2020)

44. Yasuda, K., Wang, X., Watanabe, K., Taniguchi, T. \& Jarillo-Herrero, P. Stacking-engineered ferroelectricity in bilayer boron nitride. arXiv 2010.06600. Preprint at https://arxiv.org/abs/2010.06600 (2020).

45. Woods, C. R. et al. Commensurate-incommensurate transition in graphene on hexagonal boron nitride. Nat. Phys. 10, 451-456 (2014).

46. Chen, $\mathrm{X}$. et al. Moiré engineering of electronic phenomena in correlated oxides. Nat. Phys. 16, 631-635 (2020).

47. Xian, L., Kennes, D. M., Tancogne-Dejean, N., Altarelli, M. \& Rubio, A. Multiflat bands and strong correlations in twisted bilayer boron nitride: doping-induced correlated insulator and superconductor. Nano Lett. 19, 4934-4940 (2019).

48. Kennes, D. M., Xian, L., Claassen, M. \& Rubio, A. One-dimensional flat bands in twisted bilayer germanium selenide. Nat. Commun. 11, 1124 (2020).

49. Mata, R. A. \& Suhm, M. A. Benchmarking Quantum Chemical Methods: Are We Heading in the Right Direction? Angew. Chem. Int. Ed. 56, 11011-11018 (2017).

50. Edelberg, D. et al. Approaching the intrinsic limit in transition metal diselenides via point defect control. Nano Lett. 19, 4371-4379 (2019).

51. Huang, Y. et al. Reliable exfoliation of large-area high-quality flakes of graphene and other two-dimensional materials. ACS Nano 9, 10612-10620 (2015).

52. Wang, L. et al. One-dimensional electrical contact to a two-dimensional material. Science 342, 614-617 (2013).

53. Kim, K. et al. Van der Waals heterostructures with high accuracy rotational alignment. Nano Lett. 16, 1989-1995 (2016).

54. Li, H. et al. Electrode-free anodic oxidation nanolithography of lowdimensional materials. Nano Lett. 18, 8011-8015 (2018).

55. Saito, Y., Ge, J., Watanabe, K., Taniguchi, T. \& Young, A. F. Independent superconductors and correlated insulators in twisted bilayer graphene. Nat. Phys. 16, 926-930 (2020).

56. Girit, C. O. \& Zettl, A. Soldering to a single atomic layer. Appl. Phys. Lett. 91, 193512 (2007)
57. Sunku, S. S. et al. Nano-photocurrent mapping of local electronic structure in twisted bilayer graphene. Nano Lett. 20, 2958-2964 (2020).

58. Kresse, G. \& Furthmüller, J. Efficient iterative schemes for ab initio totalenergy calculations using a plane-wave basis set. Phys. Rev. B 54, 11169-11186 (1996).

59. Blöchl, P. E. Projector augmented-wave method. Phys. Rev. B 50, 17953-17979 (1994).

60. Kresse, G. \& Joubert, D. From ultrasoft pseudopotentials to the projector augmented-wave method. Phys. Rev. B 59, 1758-1775 (1999).

61. Perdew, J. P., Burke, K. \& Ernzerhof, M. Generalized gradient approximation made simple. Phys. Rev. Lett. 77, 3865-3868 (1996).

62. Grimme, S. Semiempirical GGA-Type Density Functional Constructed with a Long-Range Dispersion Correction. J. Comput. Chem. 27, 1787-1799 (2006).

63. Tkatchenko, A. \& Scheffler, M. Accurate molecular van der Waals interactions from ground-state electron density and free-atom reference data. Phys. Rev. Lett. 102, 073005 (2009).

64. Dion, M., Rydberg, H., Schröder, E., Langreth, D. C. \& Lundqvist, B. I. Van der Waals density functional for general geometries. Phys. Rev. Lett. 92, 246401 (2004).

65. Klimeš, J., Bowler, D. R. \& Michaelides, A. Van der Waals density functionals applied to solids. Phys. Rev. B 83, 195131 (2011).

66. Klimeš, J., Bowler, D. R. \& Michaelides, A. Chemical accuracy for the van der Waals density functional. J. Phys. Condens. Matter 22, 022201 (2010).

67. Grimme, S., Antony, J., Ehrlich, S. \& Krieg, H. A consistent and accurate ab initio parametrization of density functional dispersion correction (DFT-D) for the 94 elements H-Pu. J. Chem. Phys. 132, 154104 (2010)

\section{Acknowledgements}

Research at Columbia on moiré superlattices is supported as part of Programmable Quantum Materials, an Energy Frontier Research Center funded by the U.S. Department of Energy (DOE), Office of Science, Basic Energy Sciences (BES), under award DESC0019443. STM instrumentation for STM experiments was developed with support from the Air Force Office of Scientific Research via grant FA9550-16-1-0601. Synthesis of $\mathrm{MoSe}_{2}$ and $\mathrm{WSe}_{2}$ crystals was supported by the NSF MRSEC program through Columbia in the Center for Precision Assembly of Superstratic and Superatomic Solids (DMR2011738). A.R. acknowledges support by the European Research Council (ERC-2015AdG-694097), Grupos Consolidados (IT1249-19), SFB925 and the Flatiron Institute, a division of the Simons Foundation. We acknowledge funding by the Deutsche Forschungsgemeinschaft (DFG) under Germany's Excellence Strategy - Cluster of Excellence Matter and Light for Quantum Computing (ML4Q) EXC 2004/1-390534769, funding by Advanced Imaging of Matter (AIM) EXC 2056-390715994, funding by the DFG under RTG 1995 and RTG 2247 and by DFG within the Priority Program SPP 2244 "2DMP". We acknowledge support from the Max Planck-New York City Center for Non-Equilibrium Quantum Phenomena. Work at Harvard was supported by the STC Center for Integrated Quantum Materials NSF Grant No. DMR1231319 and ARO MURI Award No. W911NF-14-0247. D.H. was supported by a grant from the Simons Foundation (579913). N.R.F. acknowledges support from the Stewardship Science Graduate Fellowship program provided under cooperative agreement number DE-NA0003864. C.R.V. acknowledges funding from the European Union's Horizon 2020 research and innovation programme under the Marie Skłodowska-Curie grant agreement No 844271 D.N.B. is the Vannevar Bush Faculty Fellow ONR-VB: N00014-19-1-263 and the Moore Investigator in Quantum Materials EPIQS \#9455.

\section{Author contributions}

D.H. conceived the moiré metrology framework, developed the relaxation codes and performed related analysis. S. Chen and D.G.A. prepared the TBG device with supervision by J.C.H. and C.R.D. N.R.F. and C.Z. fabricated all TDBG devices for nano-optics experiments with supervision by J.C.H. and C.R.D. L.W. fabricated TDBG devices for STM experiments with supervision by J.C.H. S.S. fabricated T-H-MoSe $e_{2} / \mathrm{WSe}_{2}$ devices and performed their STM study with supervision by A.N.P. D.R. grew the $\mathrm{MoSe}_{2}$ and $\mathrm{WSe}_{2}$ crystals with supervision by J.C.H. K.W. and T.T. grew the hBN crystals. Cryogenic nano-photocurrent imaging of TBG was done by D.H. and S.S.S. with assistance from A.S.M. and supervision by D.N.B. D.H. performed s-SNOM imaging of TDBG with supervision by D.N.B. A.K. and C.R.V. performed and analyzed the STM/STS measurements of TDBG with supervision by A.N.P. L.X. performed DFT calculations for GSFE of TDBG with supervision by A.R. and S.Carr performed DFT calculations for GSFE of $\mathrm{MoSe}_{2} / \mathrm{WSe}_{2}$ with supervision by E.K. Manuscript was written by D.H. and D.N.B. with contributions by D.M.K., N.R.F., S.Carr, A.S.M., and A.R. All authors contributed by discussions with particular contribution with interpretation of theory by D.M.K, S.Carr and A.R.

\section{Competing interests}

The authors declare no competing interests. 


\section{Additional information}

Supplementary information is available for this paper at https://doi.org/10.1038/s41467020-20428-1.

Correspondence and requests for materials should be addressed to D.H.

Peer review information Nature Communications thanks Andres Castellanos-Gomez, Jacek Majewski and the other anonymous reviewer(s) for their contribution to the peer review of this work. Peer reviewer reports are available.

Reprints and permission information is available at http://www.nature.com/reprints

Publisher's note Springer Nature remains neutral with regard to jurisdictional claims in published maps and institutional affiliations. (c) (i) Open Access This article is licensed under a Creative Commons Attribution 4.0 International License, which permits use, sharing, adaptation, distribution and reproduction in any medium or format, as long as you give appropriate credit to the original author(s) and the source, provide a link to the Creative Commons license, and indicate if changes were made. The images or other third party material in this article are included in the article's Creative Commons license, unless indicated otherwise in a credit line to the material. If material is not included in the article's Creative Commons license and your intended use is not permitted by statutory regulation or exceeds the permitted use, you will need to obtain permission directly from the copyright holder. To view a copy of this license, visit http://creativecommons.org/ licenses/by/4.0/.

(C) The Author(s) 2021 